Supporting Information for

\title{
Nanobubble Labeling and Imaging with a Solvatochromic Fluorophore Nile Red
}

Zhuoyu Peng and Bo Zhang*

Department of Chemistry, University of Washington, Seattle, Washington 98195 United States

Corresponding author, zhangb@uw.edu

Phone: 2065431767

\section{Table of Contents}

Video S1: Nanobubble labeling and imaging with $20 \mathrm{nM}$ Nile red.

Figure S1: Control experiments for solutions containing acetone.

Figure S2: Nanobubble labeling/imaging with R6G in a potential scan experiment.

Figure S3: Potential-dependent nanobubble labeling with Nile red.

Figure S4: Intensity and duration comparisons for nanobubble labeling events using Nile red and R6G.

Figure S5: Nanobubble labeling/imaging with $5 \mathrm{nM}$ Nile red and R6G at -1 V.

Figure S6. Multi-fluorophore nanobubble labeling of $5 \mathrm{nM}$ Nile red.

Figure S7. Fluorescence intensity-time traces for nanobubbles labeling events using $15 \mathrm{nM}$ Nile red.

Video S1: Imaging nanobubbles during a potential scan (0 to -2 to $0 \mathrm{~V}$ vs Pt QRE at $100 \mathrm{mV} / \mathrm{s}$ ) in an aqueous solution containing $20 \mathrm{nM}$ Nile red and $1 \mathrm{M} \mathrm{Na}_{2} \mathrm{SO}_{4}$. Scale bar is $5 \mu \mathrm{m}$. 
Figure S1. (A) FEEM image selected from an experiment with $1 \mathrm{M} \mathrm{Na}_{2} \mathrm{SO}_{4}$ at a constant $-1 \mathrm{~V}$ potential. (B) FEEM image selected from an experiment with $1 \mathrm{M} \mathrm{Na}_{2} \mathrm{SO}_{4}$ and $16.7 \%$ acetone at a constant $-1 \mathrm{~V}$ potential. (C) A series of TIRF images of a $22.8 \times 22.8-\mu \mathrm{m}^{2}$ area on an ITO electrode measured from a cyclic voltammetry scan from $0 \mathrm{~V}$ to $-2 \mathrm{~V}$ at $100 \mathrm{mV} / \mathrm{s}$ vs. Pt QRE containing $1 \mathrm{M} \mathrm{Na}_{2} \mathrm{SO}_{4}$ with $1 \% \mathrm{v} / \mathrm{v}$ acetone. (Scale bar: $5 \mu \mathrm{m}$ ). Video was recorded at $0.05 \mathrm{~s}$ exposure time (Frame rate: $19.81 \mathrm{~Hz}$ ).

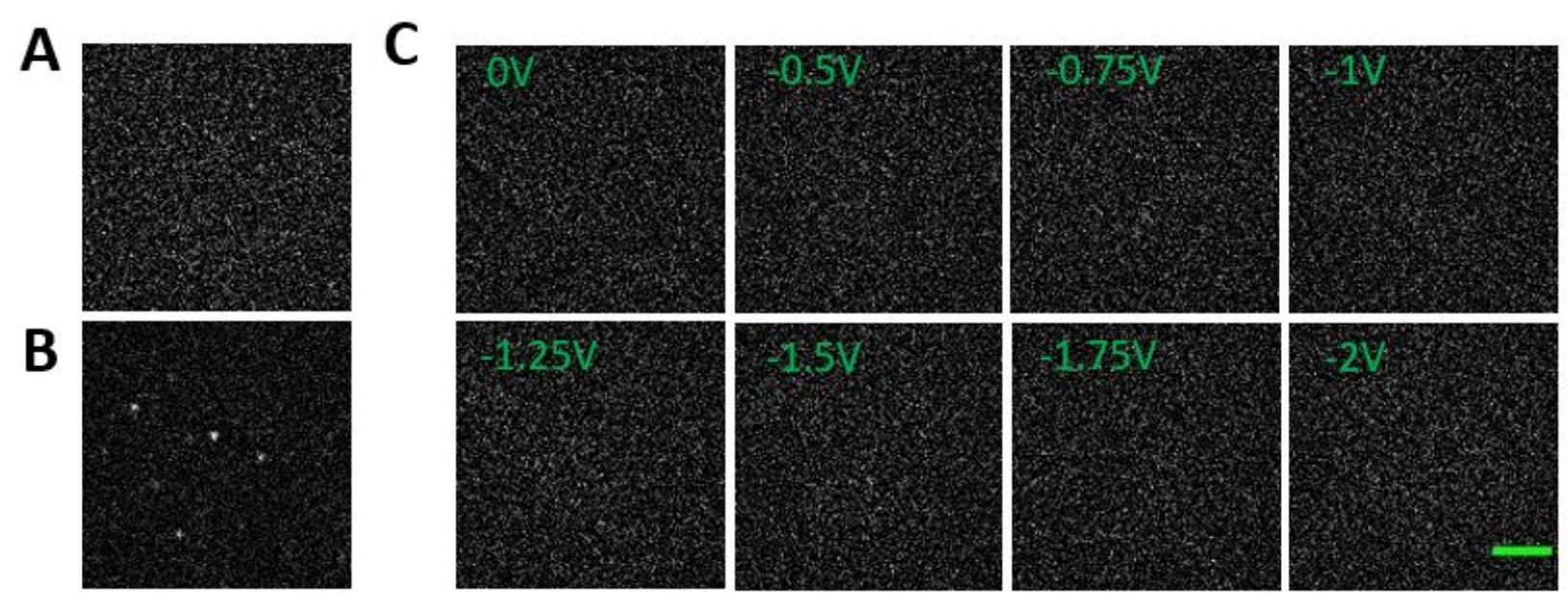

With only $1 \mathrm{M} \mathrm{Na}_{2} \mathrm{SO}_{4}$, under a constant $-1 \mathrm{~V}$ potential, no fluorescent spots can be detected. It indicates that pure electrolyte solution is free of fluorescent contaminants. (Figure S1 A)

As an organic dye, Nile red readily dissolves in more nonpolar solvents. To proceed with water reduction, Nile red needs to be dissolved in water finally. Acetone is an ideal solvent that can serve both functions: Nile red can dissolve in acetone and acetone can be miscible with water. With $16.7 \% \mathrm{v} / \mathrm{v}$ acetone in water containing $1 \mathrm{M} \mathrm{Na}_{2} \mathrm{SO}_{4}$, fluorescent contaminants are introduced (Figure S1 B). Under a constant -1V condition, $\mathrm{H}_{2}$ bubbles are generated and are labeled by contaminants. To avoid introducing fluorescent contaminants, decreasing acetone ratio would help. With $1 \% \mathrm{v} / \mathrm{v}$ acetone, no fluorescent spots can be observed, suggesting that $1 \%$ acetone is free of fluorescent contaminants (Figure S1 C) 
Figure S2. (A) The rate of nanobubble detection (detections per frame) vs potential from a potential scan from $0 \mathrm{~V}$ to $-2.0 \mathrm{~V}$ at $100 \mathrm{mV} / \mathrm{s}$ vs. Pt QRE in water containing $1 \mathrm{M} \mathrm{Na}_{2} \mathrm{SO}_{4}$ and $5 \mathrm{nM} \mathrm{R} 6 \mathrm{G}$ with $1 \%$ acetone. (B) A scatter plot showing the fluorescence intensity (counts) of individual $\mathrm{H}_{2}$ nanobubble detection events in the potential range of interest from $-0.5 \mathrm{~V}$ to $-2.0 \mathrm{~V}$ from the same recording. The photon counts of each fluorescent burst on one frame were converted from the total integrated fluorescence signal under the fitted 2D Gaussian function using ThunderSTORM.

A

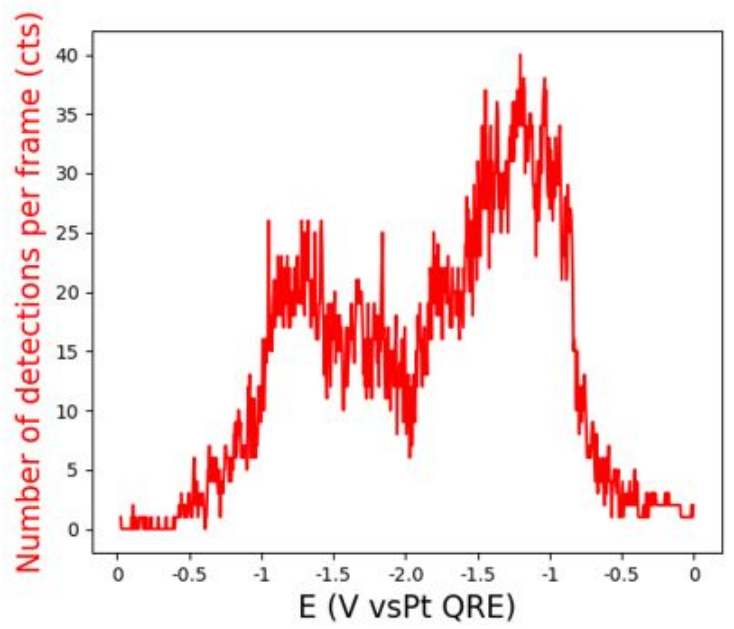

B




Figure S3. Bubbles are labeled with more fluorophores and higher frequency at a more negative potential. Upper panel: four labeling frequency plots at each voltage segments. Each spot is a $2 \times 2$ pixel region, with color representing labeling frequency. The maximum frequency is 40 frames, meaning that bubbles are labeled at all frames in this voltage segments. Lower panel: Scatter plots showing the accumulated spatial distribution of $\mathrm{H}_{2}$ nanobubbles with labeling intensity, represented by color and size. The larger and redder of the spot, the higher intensity is. Images were recorded from a voltage scan at $100 \mathrm{mV} / \mathrm{s}$ from 0 to $-2 \mathrm{~V}$ vs. Pt QRE in an aqueous solution containing $1 \mathrm{M} \mathrm{Na}_{2} \mathrm{SO}_{4}$ and $20 \mathrm{nM}$ Nile red with $1 \% \mathrm{v} / \mathrm{v}$ acetone. Video was recorded at $50 \mathrm{~ms}$ exposure time (frame rate: $19.81 \mathrm{~Hz}$ ).
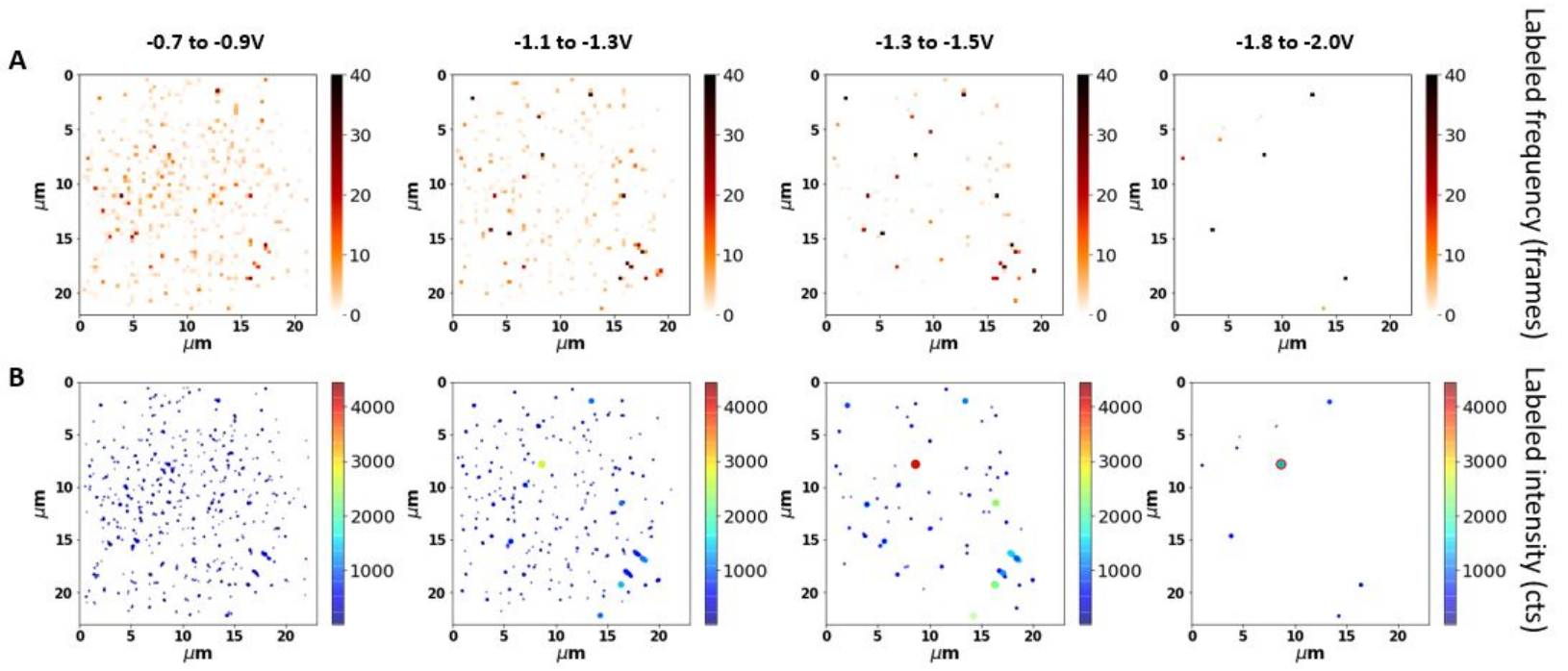
Figure S4. Intensity and duration time comparisons between $5 \mathrm{nM}$ Nile red and R6G. (A) Intensity comparison box plot of $5 \mathrm{nM}$ Nile red and R6G. Nanobubble events are collected under a constant $-1 \mathrm{~V}$ applied potential (vs Pt QRE) in solutions containing $5 \mathrm{nM}$ Nile red and $5 \mathrm{nM}$ R6G with $1 \mathrm{M} \mathrm{Na}_{2} \mathrm{SO}_{4}$ electrolyte. The horizontal and vertical lines in each box represent the median and the $25^{\text {th }}-75^{\text {th }}$ percentiles, respectively; black dots represent outlier values. (B) Histogram of the duration of nanobubble detection events. Solid lines are the single-exponential decay fits with time constants for Nile red and R6G: $138.3 \pm$ $9.1 \mathrm{~ms}$ and $106.2 \pm 3.8 \mathrm{~ms}$.

A

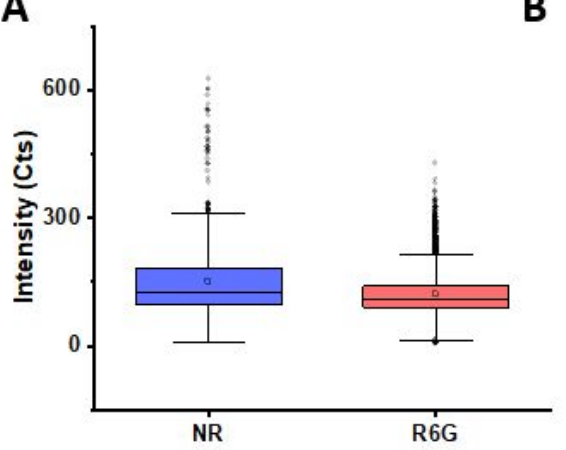

B

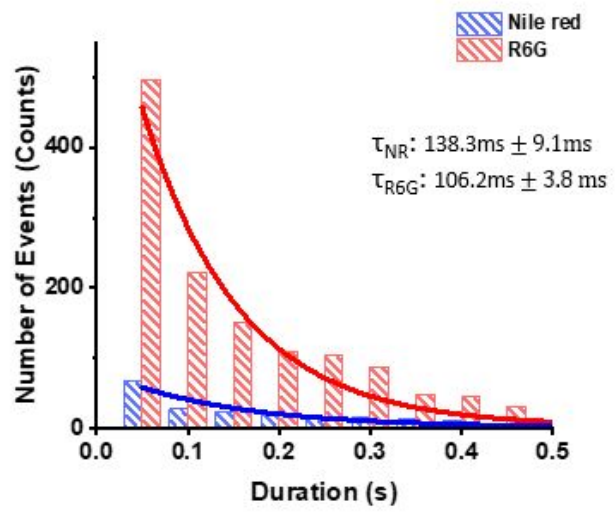


Figure S5. (A) Fluorescence intensity-time traces for $5 \mathrm{nM}$ Nile red (blue) and R6G (red) at $-1 \mathrm{~V}$. Traces were obtained by averaging the total intensity of a $6 \times 6$ pixel area around the center spot. To accurately only count the region of interest, R6G traces were obtained by averaging the total intensity of a $6 \times 6$ pixel area around the center spot. (B) Labeling frequency plots for $5 \mathrm{nM}$ Nile red (blue) and R6G (red) at $-1 \mathrm{~V}$ for total 600 frames.
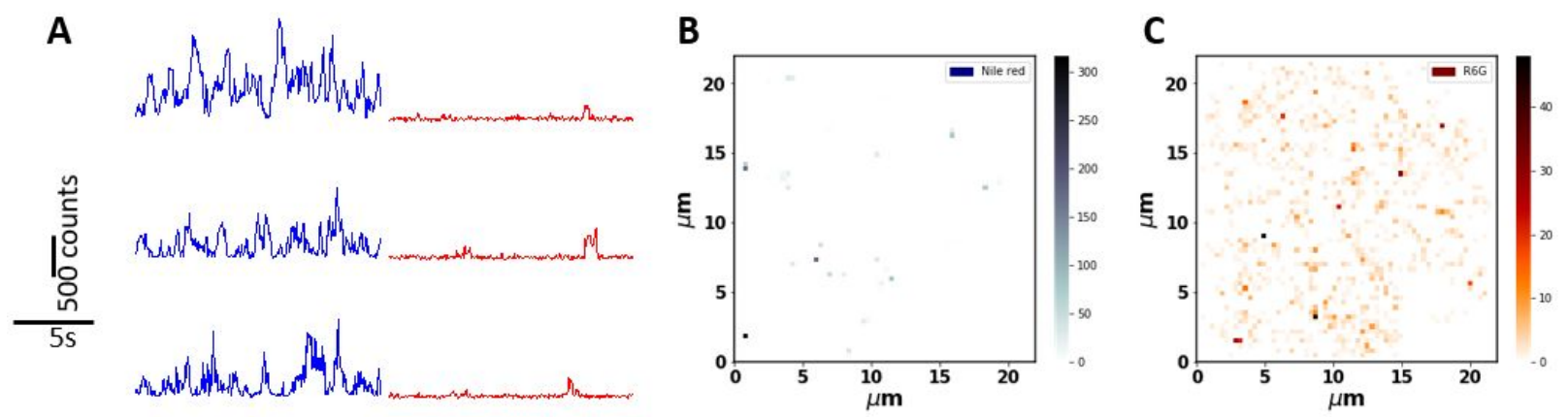
Figure S6. Multi-fluorophore nanobubble labeling of Nile red. (A) A TIRF image taken at -1.0 V showing individual $\mathrm{H}_{2}$ nanobubbles labeled by Nile red molecules. The locations of the four nanobubbles marked with color boxed, whose intensity traces are showing in (B) and (C). Images were taken in $5 \mathrm{nM}$ Nile red and $1 \mathrm{M} \mathrm{Na}_{2} \mathrm{SO}_{4}$. (Scale bar, $5 \mu \mathrm{m}$.) Images were recorded at 19.8 frames/s with a $50-\mathrm{ms}$ exposure time. In panel (B), the applied voltage was $0 \mathrm{~V}$ from the 1 st to 500 th frames and $-1.0 \mathrm{~V}$ from the 501 st to the 3000th frames. Panel (C) gives a zoom-in view of the grey shaded area in (B). Dashed blue lines were added to guide the eyes for the discrete stepwise increased in intensity. Arrows 1 to 5 mark the time points of the fluorescence images given in panel (D), which displays the fluorescence images of the same nanobubble during a three-step fluorophore labeling.

A
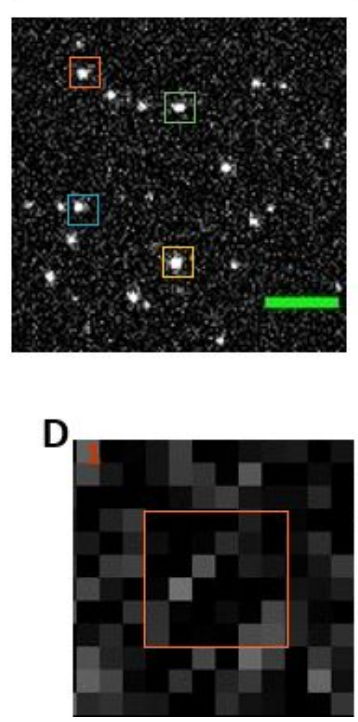
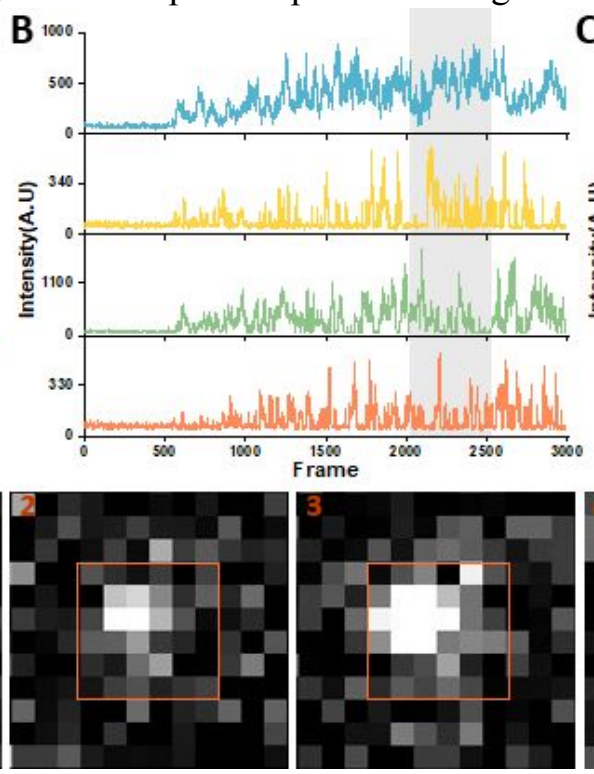
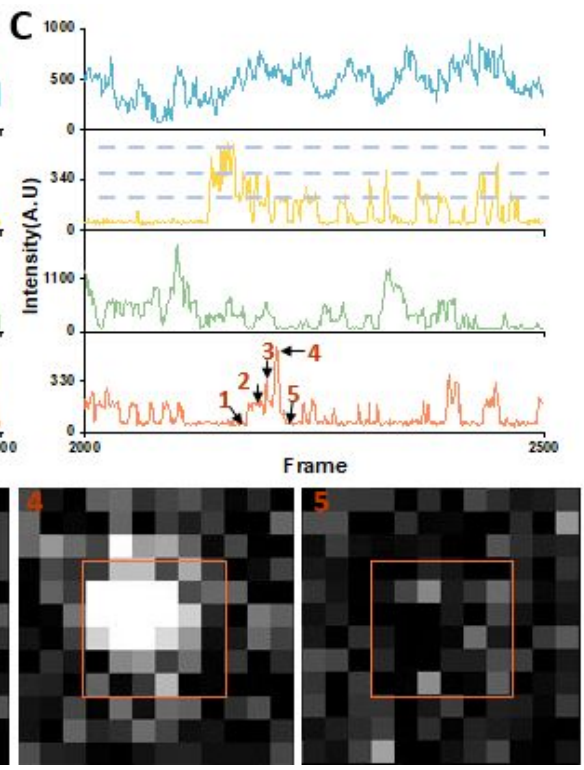
Figure S7. Fluorescence intensity-time traces for nanobubbles labeling events using $15 \mathrm{nM}$ Nile red. In panel (A), the applied voltage was at $0 \mathrm{~V}$ for the first $5 \mathrm{~s}$ and at $-1 \mathrm{~V}$ for the rest time. Panels (B) and (C) are zoom in views of the two $10 \mathrm{~s}$ sessions in panel (A) after applying $-1.0 \mathrm{~V}$. Using high concentration, one can see that the nanobubbles labeling events are no long labeled by single molecules. Instead, the same nanobubbles were continuously labeled by multiple Nile red molecules in the entire time the $-1.0 \mathrm{~V}$ potential was applied.

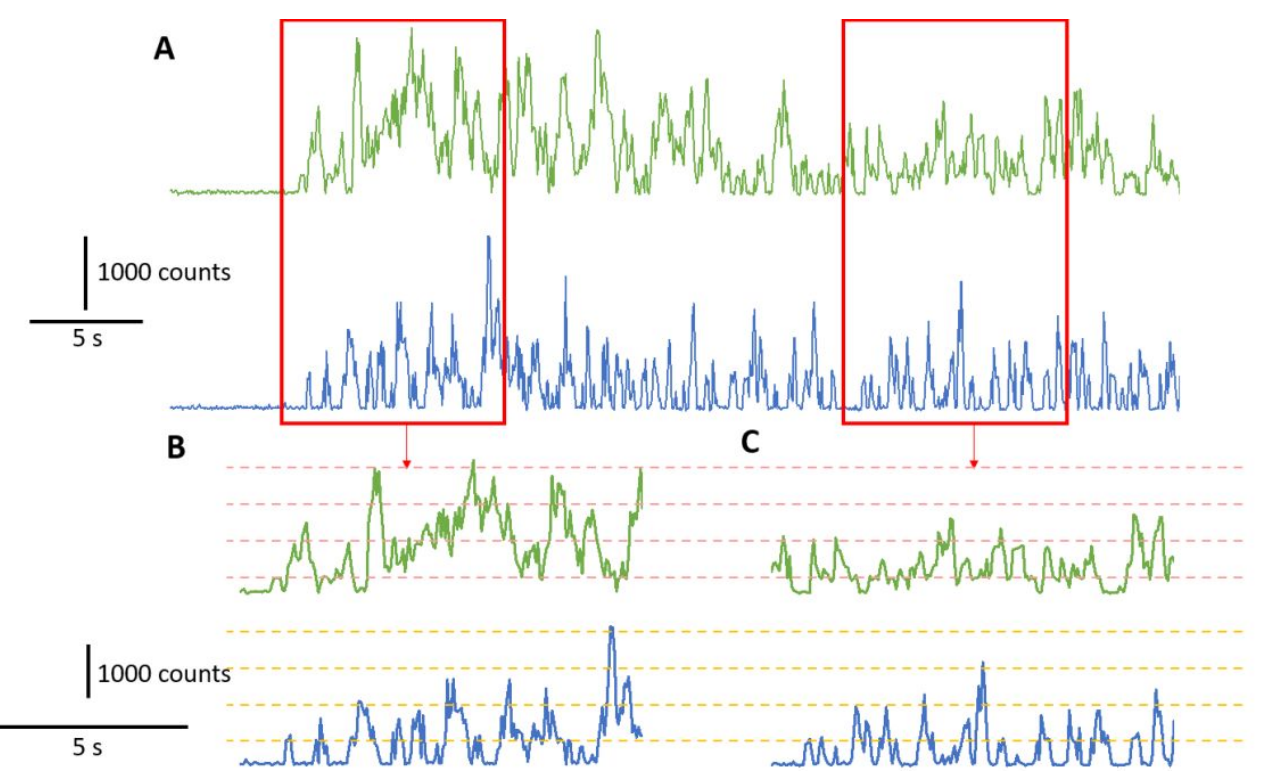

